\title{
HIV Treatment Outcomes Among Patients Initiated on Antiretroviral Therapy Pre and Post-Universal Test and Treat Guidelines in South Africa
}

This article was published in the following Dove Press journal:

Therapeutics and Clinical Risk Management

\author{
Kamban Hirasen (D' \\ Matthew P Fox ${ }^{1-3}$ \\ Cheryl J Hendrickson (D) \\ Tembeka Sineke (D) \\ Dorina Onoya $\mathbb{D}^{\prime}$
}

\begin{abstract}
'Health Economics and Epidemiology Research Office, Department of Internal Medicine, School of Clinical Medicine, Faculty of Health Sciences, University of the Witwatersrand, Johannesburg, South Africa; ${ }^{2}$ Department of Global Health, Boston University School of Public Health, Boston, MA, USA; ${ }^{3}$ Department of Epidemiology, Boston University School of Public Health, Boston, MA, USA
\end{abstract}

Introduction: Officially rolled out on 01 September 2016, South Africa's Universal Test and Treat (UTT) policy calls for first-line antiretroviral treatment (ART) initiation among all known HIV-positive patients, irrespective of CD4 cell count. We evaluate treatment outcomes of patients initiated on first-line ART directly before and after the implementation of UTT.

Methods: We analysed prospectively collected clinical cohort data among ART-naïve adult patients within two HIV clinics in Johannesburg, South Africa. We compare two groups: 1) an unexposed pre-UTT group initiating treatment from 01 December 2014 to 31 May 2015; and 2) an exposed UTT group initiating treatment from 01 December 2016 to 31 May 2017. Primary treatment outcomes included lost to follow-up (LTFU) ( $>90$ days late for the last scheduled visit with no subsequent clinical visit). Cox proportional hazards models were used to estimate the association between pre-UTT vs UTT initiation on LTFU by 12 months. Results: We included 2410 patients. A total of 1267 (52.6\%) patients initiated ART before UTT implementation and 1143 (47.4\%) after the change in policy. LTFU (adjusted Hazard Ratio (aHR): 1.51; 95\% Confidence Interval (CI): 1.16-1.98) between groups and specifically among those initiating with a CD4 cell count $\leq 500$ cells $/ \mathrm{mm}^{3}$ (aHR: $1.59 ; 95 \% \mathrm{CI}$ : 1.21-2.10) was higher among patients initiating ART under UTT.

Conclusion: LTFU under UTT proved higher than that of previous periods. Patients initiating first-line therapy under the treat-all policy may often start treatment in better health, subsequently not perceiving a direct benefit to treatment which may deter patients from consistent engagement in HIV treatment programmes.

Keywords: universal access, antiretroviral therapy, lost to follow-up, virologic suppression, resource-limited settings, South Africa

\section{Plain Language Summary}

1. Officially rolled-out in South Africa on 01 September 2016, Universal Test and Treat (UTT) calls for first-line antiretroviral therapy (ART) initiation among all known HIV-positive patients, irrespective of CD4 cell count.

2. Lost to follow-up (LTFU) was higher under UTT guidelines compared to the period directly before the shift. Patients initiating ART under UTT may often start treatment in better health, subsequently not fully perceiving a direct benefit to treatment. This coupled with the strict adherence protocol prescribed in first-line ART may deter patients from consistent engagement in HIV treatment programmes. In contrast, the need for sustained engagement with pre-ART programmes in order to initiate ART may lead to a more resilient sub-population among pre-UTT initiates.
Correspondence: Dorina Onoya Health Economics and Epidemiology Research Office, 39 Empire Road, Empire Park, Parktown, Johannesburg 2193, South Africa

Tel +27010001 7936

Email donoya@heroza.org 
3. Patients initiating first-line ART under UTT had higher virologic suppression at 12 months on treatment compared to those initiating treatment under previous guidelines. This may be attributable to relatively healthier patients initiating first-line ART under the expanded guidelines.

\section{Introduction}

The introduction of antiretroviral therapy (ART) has been crucial in reducing HIV-related morbidity and mortality as well as improving quality of life. ${ }^{1-3}$ Initiating ART treatment as soon as possible after HIV-infection has been demonstrated to reduce viral load, and decrease transmission. ${ }^{4}$ Randomized trials assessing the effects of treatment initiation at higher rather than lower CD4 cell counts have tended to focus on patients initiating ART with a CD4 cell count $<500$ cells $/ \mathrm{mm}^{3}{ }^{3-8}$ These results along with data from observational studies have provided strong evidence for the initiation of ART among patients with CD4 cell counts $>350$ cells $/ \mathrm{mm}^{3}{ }^{6}$

In 2018 there were approximately 7.5 million people living with HIV in South Africa; ${ }^{9}$ however only slightly more than half, 4.4 million (59\%) were receiving ART. ${ }^{10}$ South Africa's national ART guidelines have shifted over time towards higher initiation thresholds,${ }^{11-14}$ and in September 2016 the country implemented the World Health Organization's (WHO) recommendation of Universal Test and Treat (UTT). ${ }^{14}$ These expanded guidelines call for ART initiation of all known HIV-positive patients, irrespective of traditionally used clinical indicators/thresholds such as CD4 cell counts. ${ }^{14,15}$

Expanded guidelines are critical to getting more patients onto HIV treatment, but the increasing numbers of patients eligible for treatment could negatively impact clinics if they cannot keep up with demand. ${ }^{16}$ For providers, an increase in patient volumes may strain health-care workers and reduce quality of services, while for patients, increased access to treatment for those with higher CD4 cell counts could lead to increased attrition from care if those newly eligible patients do not see care as a priority. As South Africa progresses towards the United Nations Programme on HIV/AIDS (UNAIDS) 90-90-90 targets, ${ }^{17}$ high rates of lost to follow-up (LTFU) could reverse the considerable gains achieved by ART programmes to date. $^{16}$

We sought to add to the data on changing outcomes associated with changing guidelines by comparing 12month LTFU and virologic suppression among HIVpositive ART-naïve patients initiating first-line ART after the implementation of the UTT policy change to patients initiating treatment under the previous ART guidelines within two large public-sector HIV clinics in Johannesburg, South Africa.

\section{Methods}

\section{Study Design}

We conducted a cohort analysis of prospectively collected data (Figure 1). Our study cohort consisted of HIVpositive adult patients who initiated standard first-line triple combination ART between 01 December 2014 and 31 May 2017. Standard first-line ART during the study period consisted of the fixed-dose combination (FDC), tenofovir (TDF) with lamivudine (3TC) or emtricitabine (FTC) and efavirenz (EFV) or a combination of other standard first-line drugs when a FDC could not be prescribed. All patients were followed-up from ART initiation until 12 months post-first-line therapy.

\section{Study Population}

The study population consisted of HIV-positive nonpregnant, ART-naïve adults ( $\geq 18$ years at first-line ART initiation) without tuberculosis (TB), initiating a standard first-line ART regimen. As national test and treat algorithms differ according to pregnancy and TBinfection status, pregnant women and adults infected with TB were excluded from this analysis. ${ }^{12-14} \mathrm{We}$ compare two groups; 1) a pre-UTT (unexposed) group of all patients who initiated treatment prior to the implementation of UTT (01 December 2014-31 May 2015); and 2) an UTT (exposed) group of all patients who initiated treatment $\geq 3$ months after the implementation of UTT (01 December 2016-31 May 2017) (Figure 2) to allow the respective clinics to assimilate the change in guidelines (Figure 1). We further stratify the UTT group into those who initiated treatment at a CD4 cell count $\leq 500$ and $>500$ cells $/ \mathrm{mm}^{3}$. As per national ART guidelines during respective enrolment periods, patients enrolled during the pre-UTT period initiated ART with CD4 cell counts $\leq 500$ cells $/ \mathrm{mm}^{3}$ or with a WHO stage III/IV condition, ${ }^{12,13}$ while eligibility for ART initiation in the UTT period was based solely on patients positive HIV status. ${ }^{14}$

\section{Study Sites}

Themba Lethu HIV Clinic and Witkoppen Health and Welfare Centre are located in Johannesburg, South 


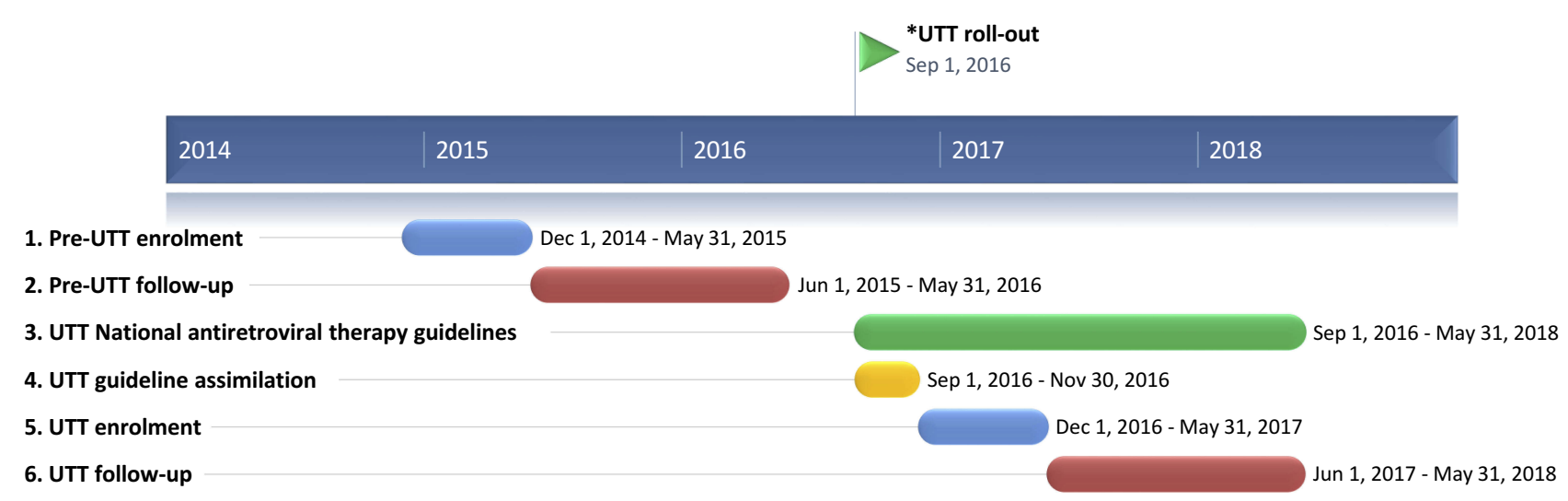

${ }^{*}$ UTT universal test and treat

1. Pre-UTT group initiated treatment from 01 December 2014 - 31 May 2015.

2. Pre-UTT group followed-up for 12 months from 01 June 2015 - 31 May 2016

3. UTT in South Africa officially rolled-out on 01 September 2016.

4. To allow the clinics to transition and implement the policy, guideline assimilation period observed from 01 September 2016 -30 November 2016.

5. UTT group initiated treatment from 01 December 2016 - 31 May 2017.

6. UTT group followed-up for 12 months from 01 June 2017 - 31 May 2018.

Figure I Cohort enrolment and follow-up among patients initiating first-line ART in South Africa Pre-UTT vs during UTT.

Africa. ${ }^{18}$ The clinics were established or expanded around 2004 as part of the public sector scale-up of ART in the country. The clinics receive technical support from Right to Care, a non-governmental organisation (NGO) funded by the United States Agency for International Development (USAID) from the President's Emergency Plan for AIDS Relief Programme (PEPFAR). While forming part of a larger clinical cohort consisting of six other clinics across two South African provinces, due to limited data availability as a result of many clinics in the cohort phasing out the longstanding existing patient management system, only the clinics listed here were considered for the analysis. ${ }^{18,19}$ HIV care and treatment within the clinics follow South African National ART Guidelines. ${ }^{11-13,20,21}$

Clinical data were captured prospectively on-site and stored in an electronic patient management system, TherapyEdgeHIV ${ }^{\text {тм}} .{ }^{19}$ Additional clinical and laboratory data were obtained from electronic records held by the National Health Laboratory Services (NHLS), the primary provider of laboratory services for South Africa's public sector clinics.

\section{Baseline Covariates}

We defined baseline as the date of first-line ART initiation among ART-naïve patients. Baseline characteristics such as age, sex and initiating ART regimen were measured at
ART initiation, while WHO stage, body mass index (BMI), and safety/work-up blood tests such as haemoglobin $(\mathrm{Hb})$ and $\mathrm{CD} 4$ cell count were measured closest to date of ART initiation (90 days before to 7 days post-ART initiation).

Due to the setting of the clinic sites (high altitude), haemoglobin levels were first adjusted down by $0.65 \mathrm{~g} /$ $\mathrm{dL}$, and defined by sex for males as 1) no anaemia $(\mathrm{Hb} \geq 13 \mathrm{~g} / \mathrm{dL}), 2)$ mild anaemia $(10<\mathrm{Hb}<13 \mathrm{~g} / \mathrm{dL}), 3)$ moderate anaemia $(8 \leq \mathrm{Hb} \leq 10 \mathrm{~g} / \mathrm{dL})$ and 4$)$ severe anaemia $(\mathrm{Hb}<8 \mathrm{~g} / \mathrm{dL})$; and for females as 1) no anaemia $(\mathrm{Hb} \geq 12 \mathrm{~g} / \mathrm{dL}), 2)$ mild anaemia $(10<\mathrm{Hb}<12 \mathrm{~g} / \mathrm{dL}), 3)$ moderate anaemia $(8 \leq \mathrm{Hb} \leq 10 \mathrm{~g} / \mathrm{dL})$ and 4$)$ severe anae$\operatorname{mia}(\mathrm{Hb}<8 \mathrm{~g} / \mathrm{dL})^{22}$

\section{Outcomes}

We compared the pre-UTT and UTT groups with respect to the following primary outcomes: 1) LTFU (defined as being $>90$ days late for the last scheduled visit with no subsequent visit) and 2) virologic suppression (defined as a viral load $\leq 400$ copies $/ \mathrm{mL}$ ) at 12 months on ART ( \pm 3 months). Secondary outcomes included missing 12 months viral load measurement among those alive and in care on ART at 12 months ( \pm 3 months). When measuring LTFU, person-time accrued from ART initiation 


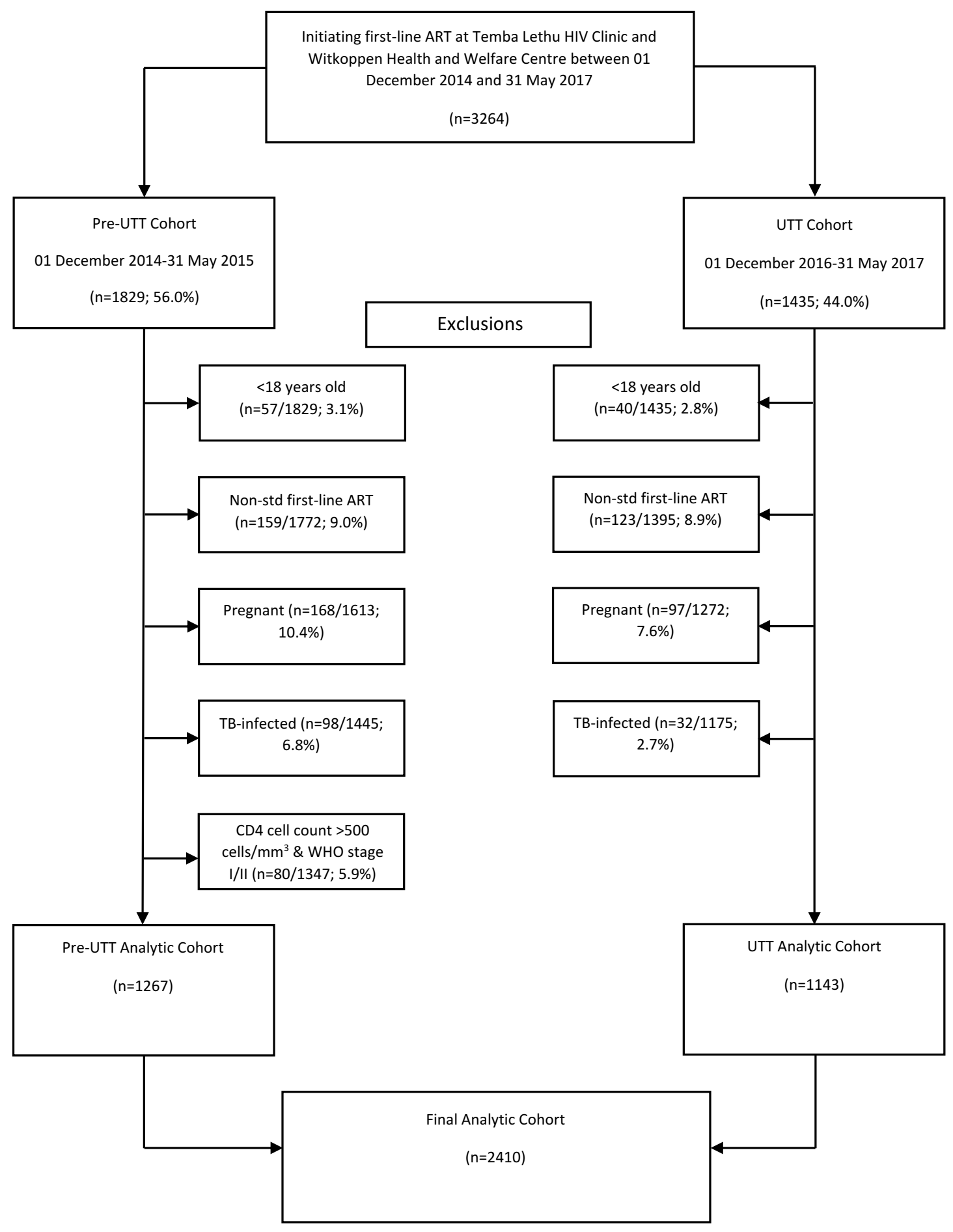

Figure 2 Cohort selection.

to the earliest of 1) LTFU; 2) death; 3) transfer out to another facility; 4) completed 12 months of follow-up. As noted, to account for some of the systematic differences between patients initiating ART across the two guideline groups, we also compare LTFU among a subpopulation of patients initiating treatment with a CD4 cell count $\leq 500$ cells $/ \mathrm{mm}^{3}$ in both groups (pre-UTT vs UTT).

\section{Statistical Analysis}

Cox proportional hazards models were used to evaluate the relationship between pre-UTT vs UTT initiation and LTFU, 
while Kaplan-Meier survival analysis was used to determine the timing of LTFU by group. Poisson regression with robust standard errors ${ }^{23}$ was used to estimate the association between pre-UTT vs UTT initiation for virologic suppression and missing viral load measurement at 12 months. All baseline characteristics know a priori to be confounders (age, sex, CD4 cell count) and/or those which resulted in a $\geq 10 \%$ change in the primary exposure effect were included in the respective adjusted models. Schoenfeld residuals were used to test the assumption of proportional hazards. All analyses presented correspond to a complete-case analysis.

\section{Ethical Approval}

All data were fully anonymised for analyses. Ethics approval for the retrospective data review was obtained from the Human Research Ethics Committee of the University of Witwatersrand (M140201) and the Boston University Institutional Review Board (H-29768).

\section{Results}

\section{Demographic and Clinical Characteristics}

We included 2410 patients in our analysis (Figure 2). Overall, 1267 (52.6\%) patients initiated ART prior to UTT implementation (01 December 2014-31 May 2015) and 1143 (47.4\%) patients initiated treatment under UTT guidelines (01 December 2016-31 May 2017). While the proportion female was similar between the pre-UTT $(60 \%)$ and UTT $\mathrm{CD} 4 \leq 500$ cells $/ \mathrm{mm}^{3}$ (55\%) groups, a higher proportion of females initiated treatment in the UTT $>500$ cells $/ \mathrm{mm}^{3}$ group (74\%) (Table 1). Median age at initiation (37 vs 37 vs 34 years) and initiating first-line regimen (96 vs 92 vs $96 \%$ on a FDC) were similar between pre-UTT and UTT patients ( $\leq 500$ vs $>500$ cells $/ \mathrm{mm}^{3}$ ) respectively. The proportion of patients diagnosed with moderate/severe anaemia varied between the pre-UTT, UTT $\leq 500$ and UTT $>500$ cells $/ \mathrm{mm}^{3}$ groups ( 21 vs 27 vs $10 \%$, respectively), while a higher proportion of pre-UTT $(25 \%)$ and UTT $\leq 500$ cells $/ \mathrm{mm}^{3}$ initiates (27\%) started ART with WHO stages III/IV conditions compared to their UTT $>500$ cells $/ \mathrm{mm}^{3}$ counterparts $(6 \%)$. Median Interquartile Range (IQR) CD4 cell count was relatively low across the pre-UTT (199 (85-325) cells $\left./ \mathrm{mm}^{3}\right)$ and UTT $\leq 500$ cells $/ \mathrm{mm}^{3}$ (189 (69-311) cells $\left./ \mathrm{mm}^{3}\right)$ groups, but increased substantially in the UTT $>500$ cells $/ \mathrm{mm}^{3}$ (673 (589-811) cells $/ \mathrm{mm}^{3}$ ) group. Low BMI $<18.5 \mathrm{~kg} / \mathrm{m}^{2}$ was similar across the pre-UTT $(11 \%)$ and UTT $\leq 500$ cells $/ \mathrm{mm}^{3}(12 \%)$ groups, but less prevalent in the UTT $>500$ cells $/ \mathrm{mm}^{3}$ (3\%) group.
By 12 months after starting ART, nearly two-thirds of all patients were still alive and in care $(66 \%)$; this was lower among UTT initiates $(\leq 500 ; 58 \%$ vs $>500$ cells $\left./ \mathrm{mm}^{3} ; 57 \%\right)$ when compared to pre-UTT initiates (75\%) (Table 2). Overall 12-months LTFU was higher among both groups of UTT initiates $(\leq 500 ; 18 \%$ vs $>500$ cells $\left./ \mathrm{mm}^{3} ; 16 \%\right)$ when compared to pre-UTT initiates $(10 \%)$. All-cause mortality was $3 \%$, reported similarly across pre-UTT and UTT $\leq 500$ cells $/ \mathrm{mm}^{3}(3 \%)$ groups but lower among the UTT $>500$ cells $/ \mathrm{mm}^{3}$ group (1\%). Transfer-out was $12 \%, 22 \%$ and $27 \%$ across the pre-UTT and UTT $(\leq 500$ vs $>500$ cells/ $\mathrm{mm}^{3}$ ) groups, respectively.

Slightly more than half $(852 / 1599 ; 53 \%)$ of those alive and in care at 12 months on ART had a 12-months viral load assessment done. The majority of these patients achieved virologic suppression at this time (79 vs 89 vs $100 \%$ among pre-UTT, UTT $\leq 500$ and UTT $>500$ cells/ $\mathrm{mm}^{3}$ respectively).

\section{LTFU by 12 Months on ART}

In adjusted models, patients initiating ART under UTT were $50 \%$ more likely to be LTFU (adjusted Hazard Ratio (aHR): 1.51; 95\% Confidence Interval (CI): 1.161.98) (Table 3). Similarly, in this population, males (aHR: 1.48; 95\% CI: $1.12-1.96)$, younger patients (18-29 vs 30-44 years; aHR: 1.49 ; 95\% CI: 1.10-2.01), and those with moderate or severe anaemia at baseline (moderate vs none; aHR: 1.65 ; 95\% CI: 1.10-2.50; severe vs none; aHR: 1.88 ; 95\% CI: $1.09-3.25)$ were approximately $50 \%$ more likely to be lost from care by 12 months after the start of ART.

\section{LTFU by 12 Months on ART - Among Patients with CD4 Cell Count $\leq 500$ cells/ $\mathrm{mm}^{3}$}

When restricted to only patients initiating treatment with a CD4 cell count $\leq 500$ cells $/ \mathrm{mm}^{3}$, those initiating under UTT guidelines were $60 \%$ more likely to be LTFU by 12 months (aHR: 1.59; 95\% CI: 1.21-2.10) (Table 4). Males (aHR: 1.56 ; 95\% CI: 1.16-2.08), younger patients (18-29 vs 30-44 years; aHR: 1.56 ; 95\% CI: 1.14-2.14), and those with moderate anaemia at baseline (moderate vs none; aHR: 1.56 ; $95 \%$ CI: $1.04-2.36)$ were also more likely to be lost from care $(56 \%)$. 
Table I Demographic and Clinical Characteristics at Treatment Initiation Among Patients Initiating ART in South Africa Pre-UTT vs During UTT

\begin{tabular}{|c|c|c|c|c|}
\hline \multirow[t]{3}{*}{ Variable } & $\begin{array}{l}\text { Pre-UTT Initiation } \\
(n=1267)\end{array}$ & $\begin{array}{l}\text { UTT Initiation CD4 } \leq 500 \\
(n=936)\end{array}$ & $\begin{array}{l}\text { UTT Initiation CD4>500 } \\
(n=207)\end{array}$ & Total $(n=2410)$ \\
\hline & $\begin{array}{l}\text { 01 December 2014-31 } \\
\text { May } 2015\end{array}$ & $\begin{array}{l}\text { 01 December 2016-31 } \\
\text { May } 2017\end{array}$ & $\begin{array}{l}\text { 01 December 2016-31 } \\
\text { May } 2017\end{array}$ & \\
\hline & n (\%) & n (\%) & n (\%) & n (\%) \\
\hline \multicolumn{5}{|l|}{ Sex } \\
\hline Female & 759 (59.9\%) & $518(55.3 \%)$ & 154 (74.4\%) & $|43|$ (59.4\%) \\
\hline \multicolumn{5}{|l|}{ Age at ART Initiation (Years) } \\
\hline Median (IQR) & $36.8(31.1-43.6)$ & $36.6(30.6-43.9)$ & $34.3(28.1-45.7)$ & $36.6(30.6-43.9)$ \\
\hline $18-29$ & $265(20.9 \%)$ & $212(22.6 \%)$ & $69(33.3 \%)$ & $546(22.7 \%)$ \\
\hline $30-44$ & $735(58.0 \%)$ & $520(55.6 \%)$ & $85(41.1 \%)$ & 1340 (55.6\%) \\
\hline $45-59$ & $234(18.5 \%)$ & $174(18.6 \%)$ & $42(20.3 \%)$ & $450(18.7 \%)$ \\
\hline$\geq 60$ & $33(2.6 \%)$ & $30(3.2 \%)$ & II (5.3\%) & $74(3.1 \%)$ \\
\hline \multicolumn{5}{|c|}{ Antiretroviral Regimen at ART Initiation } \\
\hline $\mathrm{TDF}+3 \mathrm{TC} / \mathrm{FTC}+\mathrm{EFV}$ & $1216(96.0 \%)$ & 856 (91.5\%) & $199(96.1 \%)$ & $2271(94.2 \%)$ \\
\hline $\mathrm{ABC}+3 \mathrm{TC}+\mathrm{EFV} / \mathrm{NVP}$ & $29(2.3 \%)$ & $50(5.3 \%)$ & $2(1.0 \%)$ & $81(3.4 \%)$ \\
\hline $\mathrm{TDF}+3 \mathrm{TC} / \mathrm{FTC}+\mathrm{NVP} / \mathrm{LPV} / \mathrm{r}$ & $22(1.7 \%)$ & $30(3.2 \%)$ & $6(2.9 \%)$ & $58(2.4 \%)$ \\
\hline \multicolumn{5}{|c|}{ CD4 Cell Count at ART Initiation (Cells $/ \mathrm{mm}^{3}$ ) } \\
\hline Median (IQR) & $199(85-325)$ & $189(69-311)$ & $673(589-81 \mathrm{I})$ & $224(93-369)$ \\
\hline $0-200$ & $477(50.2 \%)$ & $378(53.1 \%)$ & $0(0.0 \%)$ & $855(45.7 \%)$ \\
\hline $201-350$ & $288(30.3 \%)$ & $200(28.1 \%)$ & $0(0.0 \%)$ & $488(26.1 \%)$ \\
\hline $351-500$ & $186(19.5 \%)$ & $134(18.8 \%)$ & $0(0.0 \%)$ & $320(17.1 \%)$ \\
\hline$>500$ & $0(0.0 \%)$ & $0(0.0 \%)$ & $207(100.0 \%)$ & 207 (II.1\%) \\
\hline Missing & 316 & 224 & 0 & 540 \\
\hline \multicolumn{5}{|c|}{ Haemoglobin at ART Initiation, g/dL (Anaemia) } \\
\hline Median (IQR) & $12.5(11.0-13.8)$ & $12.0(10.5-13.5)$ & $13.5(12.0-14.5)$ & $12.4(10.9-13.8)$ \\
\hline None & $425(39.7 \%)$ & $198(33.3 \%)$ & $87(60.0 \%)$ & $710(39.2 \%)$ \\
\hline Mild & $423(39.5 \%)$ & $238(40.0 \%)$ & $44(30.3 \%)$ & $705(39.0 \%)$ \\
\hline Moderate & $167(15.6 \%)$ & $106(17.8 \%)$ & $6(4.1 \%)$ & $279(15.4 \%)$ \\
\hline Severe & $55(5.1 \%)$ & $53(8.9 \%)$ & $8(5.5 \%)$ & 116 (6.4\%) \\
\hline Missing & 197 & 341 & 62 & 600 \\
\hline \multicolumn{5}{|l|}{ WHO Stage at ART Initiation } \\
\hline 1 & $243(54.4 \%)$ & $|8|(62.0 \%)$ & $44(80.0 \%)$ & $468(58.9 \%)$ \\
\hline II & 91 (20.4\%) & $40(13.7 \%)$ & $8(14.5 \%)$ & 139 (I7.5\%) \\
\hline III & 68 (I5.2\%) & 24 (8.2\%) & $3(5.5 \%)$ & 95 (I2.0\%) \\
\hline IV & $45(10.1 \%)$ & $47(16.1 \%)$ & $0(0.0 \%)$ & $92(11.6 \%)$ \\
\hline Missing & 820 & 644 & 152 & 1616 \\
\hline \multicolumn{5}{|l|}{ BMI at ART Initiation $\left(\mathrm{Kg} / \mathrm{m}^{2}\right)$} \\
\hline Low $<18.5$ & $117(10.6 \%)$ & $95(12.1 \%)$ & $5(2.6 \%)$ & 217 (10.4\%) \\
\hline $18.5-24.9$ & $596(54.2 \%)$ & 411 (52.4\%) & $83(42.8 \%)$ & $1090(52.5 \%)$ \\
\hline $25-29.9$ & $242(22.0 \%)$ & $167(21.3 \%)$ & 59 (30.4\%) & $468(22.5 \%)$ \\
\hline$\geq 30$ & $144(13.1 \%)$ & $112(14.3 \%)$ & 47 (24.2\%) & $303(14.6 \%)$ \\
\hline Missing & 168 & 151 & 13 & 332 \\
\hline
\end{tabular}

Abbreviations: UTT, Universal test and treat; IQR, Interquartile range; TDF, Tenofovir; 3TC, Lamivudine; FTC, Emtricitabine; EFV, Efavirenz; ABC, Abacavir; NVP, Nevirapine; LPV/r, Lopinavir; WHO, World Health Organization; BMI, Body Mass Index.

\section{Timing of LTFU by 12 Months on ART}

The risk of LTFU was similar between UTT initiates $\left(\leq 500\right.$ vs $>500$ cells $\left./ \mathrm{mm}^{3}\right)$ in the first 6 months of treatment after which time the $\leq 500$ cells $/ \mathrm{mm}^{3}$ group had a marginally higher risk of the outcome (Figure 3). By 12 months, approximately $80 \%$ and $82 \%$ of UTT initiates $\left(\leq 500\right.$ vs $>500$ cells $\left./ \mathrm{mm}^{3}\right)$ were still alive and in care compared to $90 \%$ of pre-UTT initiates. 
Table 2 Treatment Outcomes at 12 Months on Treatment Among Patients Initiating ART in South Africa Pre-UTT vs During UTT

\begin{tabular}{|c|c|c|c|c|}
\hline \multirow[t]{3}{*}{ Variable } & $\begin{array}{l}\text { Pre-UTT Initiation } \\
(n=1267)\end{array}$ & $\begin{array}{l}\text { UTT Initiation CD4 } \leq 500 \\
(n=936)\end{array}$ & $\begin{array}{l}\text { UTT Initiation CD4>500 } \\
(n=207)\end{array}$ & Total $(n=24 \mid 0)$ \\
\hline & $\begin{array}{l}\text { O1 December 2014-31 } \\
\text { May } 2015\end{array}$ & $\begin{array}{l}01 \text { December 2016-31 } \\
\text { May } 2017\end{array}$ & $\begin{array}{l}\text { O1 December 2016-31 } \\
\text { May } 2017\end{array}$ & \\
\hline & n (\%) & n (\%) & n (\%) & n (\%) \\
\hline $\begin{array}{l}\text { Treatment Outcomes at I2 Months on ART } \\
\text { Alive in care } \\
\text { Lost to follow-up } \\
\text { Dead } \\
\text { Transferred-out }\end{array}$ & $\begin{array}{l}944(74.5 \%) \\
131(10.3 \%) \\
40(3.2 \%) \\
152(12.0 \%)\end{array}$ & $\begin{array}{l}538(57.5 \%) \\
164(17.5 \%) \\
29(3.1 \%) \\
205(21.9 \%)\end{array}$ & $\begin{array}{l}117(56.5 \%) \\
33(15.9 \%) \\
\text { I (0.5\%) } \\
56(27.1 \%)\end{array}$ & $\begin{array}{l}1599(66.4 \%) \\
328(13.6 \%) \\
70(2.9 \%) \\
413(17.1 \%)\end{array}$ \\
\hline $\begin{array}{l}\text { Viral Load Measurement at } 12 \text { Months on ART } \\
\text { Yes } \\
\text { No }\end{array}$ & $\begin{array}{l}505(53.5 \%) \\
439(46.5 \%)\end{array}$ & $\begin{array}{l}297(55.2 \%) \\
24 \mid(44.8 \%)\end{array}$ & $\begin{array}{l}50(42.7 \%) \\
67(57.3 \%)\end{array}$ & $\begin{array}{l}852(53.3 \%) \\
747(46.7 \%)\end{array}$ \\
\hline $\begin{array}{l}\text { Virologic suppression at } 12 \text { months on ART } \\
\text { Suppressed } \\
\text { Unsuppressed }\end{array}$ & $\begin{array}{l}397(78.6 \%) \\
108(21.4 \%)\end{array}$ & $\begin{array}{l}265(89.2 \%) \\
32(10.8 \%)\end{array}$ & $\begin{array}{l}50(100.0 \%) \\
0(0.0 \%)\end{array}$ & $\begin{array}{l}712(83.6 \%) \\
140(16.4 \%)\end{array}$ \\
\hline
\end{tabular}

Notes: ${ }^{a}$ Among those alive and in care at 12 months $(n=1599) ;{ }^{b}$ Among patients with a viral load recorded at 12 months $( \pm 3$ months). Abbreviation: UTT, Universal test and treat.

\section{Viral Load Suppression at I 2 Months on ART}

Among those alive and in care with viral loads recorded at 12 months post-ART initiation, there was a slight improvement in virologic suppression rates after the implementation of UTT (UTT adjusted relative risk (aRR): 1.13; 95\% CI: 1.06-1.19) (Table 5). Similarly, older adults ( $\geq 60$ vs $30-44$ years; aRR: 1.12; 95\% CI: $1.05-1.19)$ and those with higher CD4 cell counts (201-350 vs 0-200; aRR: 1.15 ; $95 \%$ CI: $1.07-1.24$; $>350$ vs $0-200$ cells $/ \mathrm{mm}^{3}$; aRR: 1.17 ; 95\% CI: $1.09-1.26$ ) were more likely to achieve virologic suppression at 12 months on treatment.

\section{Missing Viral Load at 12 Months on ART}

Of those alive and in care at 12 months on ART, almost half $(46.7 \%$; 747/1599) were missing a viral load measurement. This was similar between those initiating ART under UTT guidelines $(47.0 \%$; 308/655), and those initiating treatment prior to the guideline change $(46.5 \%$; 439/944). Males (aRR: 1.18; 95\% CI: 1.03-1.35) and patients with higher CD4 cell counts (>350 vs $0-200$ cells $/ \mathrm{mm}^{3}$; aRR: $1.21 ; 95 \%$ CI: $1.03-1.42$ ) were at least $20 \%$ more likely to have a missing viral load at this time.

\section{Discussion}

Treatment outcomes such as LTFU and virologic suppression pre and post recent universal test and treat guidelines have currently not been evaluated in routine care in South Africa. In this study, LTFU by 12 months on first-line ART was $13.6 \%$. This falls within the range (3.3-25.0\%) commonly observed in the first year of sub-Saharan African HIV treatment programmes. ${ }^{24,25}$ Patients initiating ART under UTT guidelines (initiating treatment irrespective of CD4 cell count or WHO stage) were 51\% (aHR: 1.51; 95\% CI: 1.16-1.98) more likely to be lost from care by 12 months when compared to those initiating ART prior to the implementation of the policy (initiating treatment based on CD4 cell count $\leq 500 \mathrm{cell} / \mathrm{mm}^{3} / \mathrm{WHO}$ stage III/ IV condition). Similarly, when restricted to only patients initiating treatment with a CD4 cell count $\leq 500 \mathrm{cells} / \mathrm{mm}^{3}$, patients initiating under UTT guidelines were 59\% (aHR: 1.59; 95\% CI: $1.21-2.10$ ) more likely to be lost from care. While common risk factors of LTFU include; dissatisfaction with health services, financial constraints, poor infrastructure surrounding health facilities and adverse effects of treatment, potential precursors to the outcome also include, improving health condition and higher initiating CD4 cell count. ${ }^{16,26-31}$ The newly implemented treatment policy has resulted in patients initiating ART at increasingly better health status (moderately higher CD4 cell count in this study). These patients may not fully perceive the direct benefits of treatment, particularly in the context of travel time and transport costs to the clinic, long queues and waiting times. Additionally, first-line ART is prescribed with a strict adherence protocol. ${ }^{32}$ The perceived non-benefit of treatment in addition to the health system barriers to easy access to HIV care may result in increased 
Table 3 Unadjusted and Adjusted Estimates of the Relation Between Pre-UTT Initiation and UTT Initiation on LTFU

\begin{tabular}{|c|c|c|c|}
\hline \multirow[t]{2}{*}{ Variable } & \multirow[t]{2}{*}{ n (\%) } & \multicolumn{2}{|c|}{$\begin{array}{l}\text { LTFU by } 12 \text { Months } \\
(n=328 / 24 \mid 0)\end{array}$} \\
\hline & & $\begin{array}{l}\text { Crude HR } \\
(95 \% \mathrm{Cl})\end{array}$ & $\begin{array}{l}\text { Adjusted HR } \\
(95 \% \mathrm{Cl})^{\mathrm{a}}\end{array}$ \\
\hline $\begin{array}{l}\text { UTT Status } \\
\text { Pre-UTT } \\
\text { UTT }\end{array}$ & $\begin{array}{l}131 / 1267(10.3 \%) \\
197 / 1143(17.2 \%)\end{array}$ & $\begin{array}{l}1.00 \\
1.84(1.48-2.30)\end{array}$ & $\begin{array}{l}1.00 \\
1.51(1.16-1.98)\end{array}$ \\
\hline $\begin{array}{l}\text { Sex } \\
\text { Female } \\
\text { Male }\end{array}$ & $\begin{array}{l}176 / 1431(12.3 \%) \\
152 / 979(15.5 \%)\end{array}$ & $\begin{array}{l}1.00 \\
1.28(1.03-1.59)\end{array}$ & $\begin{array}{l}1.00 \\
1.48(1.12-1.96)\end{array}$ \\
\hline $\begin{array}{l}\text { Age at ART } \\
\text { Initiation (Years) } \\
\begin{array}{l}18-29 \\
30-44 \\
44-59 \\
\geq 60\end{array}\end{array}$ & $\begin{array}{l}97 / 546(17.8 \%) \\
172 / 1340(12.8 \%) \\
47 / 450(10.4 \%) \\
12 / 74(16.2 \%)\end{array}$ & $\begin{array}{l}1.46(1.13-1.87) \\
1.00 \\
0.81(059-1.12) \\
1.28(0.72-2.31)\end{array}$ & $\begin{array}{l}1.49(1.10-2.01) \\
1.00 \\
0.77(0.52-1.14) \\
1.29(0.63-2.66)\end{array}$ \\
\hline $\begin{array}{l}\text { CD4 Cell Count } \\
\text { at ART Initiation } \\
\left(\text { Cells } / \mathrm{mm}^{3}\right) \\
0-200 \\
201-350 \\
>350\end{array}$ & $\begin{array}{l}126 / 855(14.7 \%) \\
67 / 488(13.7 \%) \\
88 / 527(16.7 \%)\end{array}$ & $\begin{array}{l}1.00 \\
0.91(0.67-1.22) \\
1.10(0.84-1.45)\end{array}$ & $\begin{array}{l}1.00 \\
0.98(0.70-1.38) \\
1.10(0.78-1.54)\end{array}$ \\
\hline $\begin{array}{l}\text { Anaemia at ART } \\
\text { Initiation } \\
\text { None } \\
\text { Mild } \\
\text { Moderate } \\
\text { Severe }\end{array}$ & $\begin{array}{l}88 / 710(12.4 \%) \\
102 / 705(14.5 \%) \\
44 / 279(15.8 \%) \\
19 / 116(16.4 \%)\end{array}$ & $\begin{array}{l}1.00 \\
1.22(0.92-1.62) \\
1.41(0.98-2.02) \\
1.62(0.99-2.66)\end{array}$ & $\begin{array}{l}1.00 \\
1.37(0.99-1.88) \\
1.65(1.10-2.50) \\
1.88(1.09-3.25)\end{array}$ \\
\hline
\end{tabular}

Notes: ${ }^{a}$ Model adjusted for the following variables at ART initiation: UTT status, sex, age, CD4 cell count and anaemia. Schoenfeld residuals $(p=0.503$; proportionality assumption satisfied).

Abbreviations: LTFU, Lost to follow-up; HR, Hazard ratio; UTT, Universal test and treat.

defaulter rates after ART initiation. Important to consider, patients initiating ART under UTT guidelines are eligible for treatment upon positive testing. Patients from this group who go on to initiate treatment, may not be subjected to the same pre-initiation protocols/procedures as those who initiate under pre-UTT guidelines who become eligible (CD4 $\leq 500$ cells $/ \mathrm{mm}^{3}$ ) sometime after testing positive with a CD4 cell count $>500$ cells $/ \mathrm{mm}^{3}$. The latter group, may then be more resilient once on treatment due to their sustained retention in pre-ART programmes.

Other risk factors of LTFU by 12 months on ART in this population ( $\geq 50 \%$ more likely to be LTFU) included; male gender, younger adults and anaemia. As commonly observed in resource-limited HIV treatment programmes, younger patients often face many barriers in accessing and
Table 4 Unadjusted and Adjusted Estimates of the Relation Between Pre-UTT Initiation and UTT Initiation on LTFU Among Patients Initiating Treatment with a CD4 Cell Count $\leq 500$ Copies $/ \mathrm{mm}^{3}$

\begin{tabular}{|c|c|c|c|}
\hline \multirow[t]{2}{*}{ Variable } & \multicolumn{3}{|c|}{$\begin{array}{l}\text { LTFU by } 12 \text { Months }(\mathrm{n}=7 \mid \mathrm{I} / 1662) \text { - } \\
\text { CD4 Cell count } \leq 500 \text { Copies } / \mathrm{mm}^{3}\end{array}$} \\
\hline & n (\%) & $\begin{array}{l}\text { Crude HR } \\
(95 \% \mathrm{Cl})\end{array}$ & $\begin{array}{l}\text { Adjusted HR } \\
(95 \% \mathrm{Cl})^{\mathrm{a}}\end{array}$ \\
\hline \multicolumn{4}{|l|}{ UTT Status } \\
\hline Pre-UTT & |10/95| (11.6\%) & 1.00 & 1.00 \\
\hline UTT & |38/7|| (19.4\%) & $1.86(1.45-2.39)$ & $1.59(1.21-2.10)$ \\
\hline \multicolumn{4}{|l|}{ Sex } \\
\hline Female & 122/930 (13.1\%) & 1.00 & 1.00 \\
\hline Male & 126/732 (17.2\%) & $1.32(1.03-1.69)$ & $1.56(1.16-2.08)$ \\
\hline \multicolumn{4}{|l|}{ Age at ART } \\
\hline \multicolumn{4}{|c|}{ Initiation (Years) } \\
\hline $18-29$ & $71 / 371$ (19.1\%) & $1.43(1.07-1.91)$ & $1.56(1.14-2.14)$ \\
\hline $30-44$ & |33/94| (14.1\%) & 1.00 & 1.00 \\
\hline $44-59$ & $37 / 300(12.3 \%)$ & $0.86(0.60-1.23)$ & $0.79(0.53-1.19)$ \\
\hline$\geq 60$ & $7 / 50$ (14.0\%) & $1.02(0.48-2.18)$ & $1.17(0.51-2.68)$ \\
\hline \multirow{2}{*}{\multicolumn{4}{|c|}{ CD4 Cell Count }} \\
\hline & \multicolumn{3}{|c|}{ at ART Initiation } \\
\hline \multicolumn{4}{|c|}{$\left(\right.$ Cells $\left./ \mathrm{mm}^{3}\right)$} \\
\hline$\leq 350$ & $193 / 1343$ (14.4\%) & 1.00 & 1.00 \\
\hline $350-500$ & $55 / 319(17.2 \%)$ & $1.15(0.85-1.55)$ & $1.23(0.87-1.74)$ \\
\hline \multicolumn{4}{|c|}{ Anaemia at ART } \\
\hline \multicolumn{4}{|l|}{ Initiation } \\
\hline None & $68 / 519$ (13.1\%) & 1.00 & 1.00 \\
\hline Mild & $84 / 566(14.8 \%)$ & $1.18(0.86-1.62)$ & $1.26(0.91-1.74)$ \\
\hline Moderate & $39 / 237$ (16.5\%) & $1.39(0.94-2.06)$ & $1.56(1.04-2.36)$ \\
\hline Severe & 15/94 (16.0\%) & $1.50(0.86-2.62)$ & $1.68(0.95-2.99)$ \\
\hline
\end{tabular}

Notes: a Model adjusted for the following variables at ART initiation: UTT status, sex, age, CD4 cell count and anaemia. Schoenfeld residuals ( $p=0.529$; proportionality assumption satisfied).

Abbreviations: LTFU, Lost to follow-up; HR, Hazard ratio; UTT, Universal test and treat.

maintaining HIV treatment. These barriers typically come in the form of negative attitudes and perceptions held by health-care workers such as counsellors and nurses, irregular routines while pursuing tertiary education and employment as well as higher risk-taking behaviour. ${ }^{2,33-35}$ HIV-positive patients with anaemia are often subject to other opportunistic infections, complications in treatment and disease progression; common risk factors to both mortality, disengagement with health services and potentially progression to higherlevel facilities/hospitalization. ${ }^{36,37}$

Patients initiating ART under UTT and those with higher CD4 cell counts $\left(>200\right.$ cells $\left./ \mathrm{mm}^{3}\right)$ were at least $15 \%$ more likely to achieve virologic suppression at 12 months on ART. While this may be a marginal increase, the higher suppression rates among those in better health 


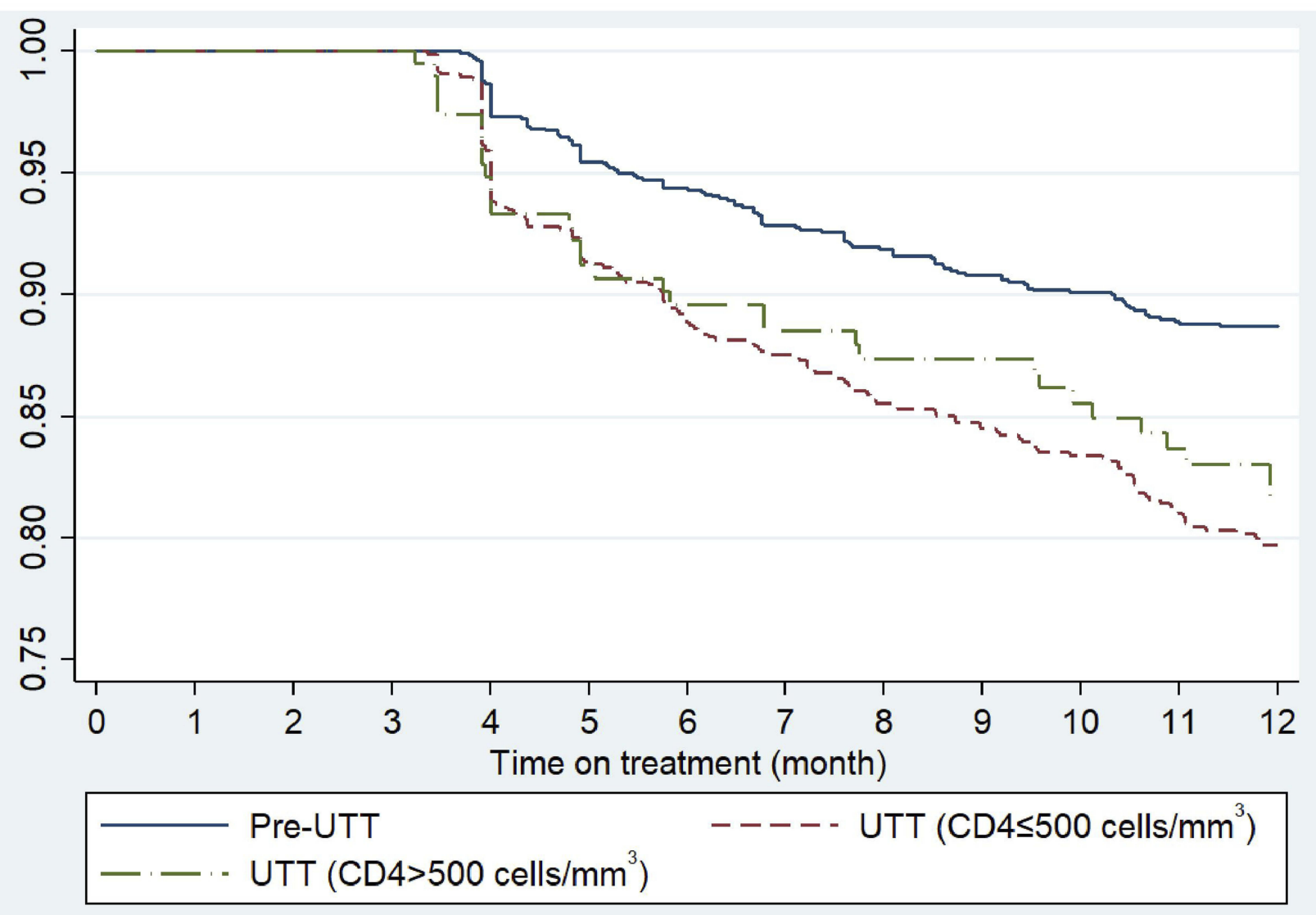

Figure 3 Kaplan-Meier survival estimates of lost to follow-up among patients initiating ART in South Africa Pre-UTT vs during UTT ( $\mathrm{n}=24 \mathrm{I0}$ ).

at the start of treatment may speak to the rationale behind earlier ART initiation (at a higher CD4 cell count). Higher CD4 cell count at ART initiation has subsequently been established as a clinical characteristic generally leading to higher rates of virologic suppression on ART. ${ }^{38}$ Higher rates of suppression among patients initiating treatment with higher CD4 cell counts may also account for the increase in missing viral load measurement among this group at 12 months on treatment. These patients, often in better health may not warrant closer viral load monitoring when compared to those with poorer virologic response early on in ART.

\section{Limitations}

Patients enrolled in the pre-UTT and UTT groups were enrolled in two mutually exclusive periods. This aspect of the study design was a function of the national treatment guideline changes between the study time periods and ensured two distinct treatment groups. While the nonoverlapping nature of our respective cohorts may allow direct comparisons of outcomes across respective periods, it may create confounding through changes or improvements in programmatic conditions between such periods. Programmatic changes (eg, tracing initiatives) could have potentially affected treatment outcomes investigated here, although we would expect such initiatives to improve over time, leading to lower rates of LTFU in the UTT period.

A lack of pre-ART data among the pre-UTT group restricts our ability to directly assess the causal effect of UTT. Differences in the pre-ART initiation protocols across respective guidelines does not allow for the populations themselves to be directly comparable. However, in order to account for differences in eligibility between groups and its effect on pre-initiation protocols and outcomes, we compare a sub-population of the UTT group (CD4 cell count $\leq 500$ cells $/ \mathrm{mm}^{3}$ ) to the pre-UTT group (CD4 cell count $\leq 500$ cells $/ \mathrm{mm}^{3}$ ) with regard to LTFU. Initiating treatment under UTT guidelines, male gender, younger age and moderate anaemia were still identified as risk factors of LTFU by 12 months on treatment. 
Table 5 Unadjusted and Adjusted Estimates of the Relation Between Pre-UTT Initiation and UTT Initiation on Virologic Suppression at 12 Months (VL $\leq 400$ Copies $/ \mathrm{mL}$ )

\begin{tabular}{|c|c|c|c|}
\hline \multirow[t]{2}{*}{ Variable } & \multirow[t]{2}{*}{ n (\%) } & \multicolumn{2}{|c|}{$\begin{array}{l}\text { Virologic Suppression at } 12 \\
\text { Months }(n=7|2 / 24| 0)\end{array}$} \\
\hline & & $\begin{array}{l}\text { Crude RR } \\
(95 \% \mathrm{Cl})\end{array}$ & $\begin{array}{l}\text { Adjusted RR } \\
(95 \% \mathrm{Cl})^{\mathrm{a}}\end{array}$ \\
\hline \multicolumn{4}{|l|}{ UTT Status } \\
\hline Pre-UTT & $397 / 505(78.6 \%)$ & 1.00 & 1.00 \\
\hline UTT & $315 / 347(90.8 \%)$ & $1.15(1.09-1.22)$ & $1.13(1.06-1.19)$ \\
\hline \multicolumn{4}{|l|}{ Sex } \\
\hline Female & $434 / 517$ (84.0\%) & 1.00 & 1.00 \\
\hline Male & $278 / 335(83.0 \%)$ & $0.99(0.93-1.05)$ & $1.01(0.95-1.08)$ \\
\hline \multicolumn{4}{|l|}{ Age at ART } \\
\hline \multicolumn{4}{|c|}{ Initiation (Years) } \\
\hline $18-29$ & $159 / 190(83.7 \%)$ & $1.03(0.96-1.12)$ & $0.97(0.90-1.05)$ \\
\hline $30-44$ & $384 / 474(81.0 \%)$ & 1.00 & 1.00 \\
\hline 44-59 & $146 / 165(88.5 \%)$ & $1.09(1.02-1.17)$ & $1.06(0.99-1.13)$ \\
\hline$\geq 60$ & $23 / 23(100.0 \%)$ & $1.23(1.18-1.29)$ & $1.12(1.05-1.19)$ \\
\hline \multicolumn{4}{|c|}{ CD4 Cell Count at } \\
\hline \multicolumn{4}{|c|}{ ART Initiation } \\
\hline \multicolumn{4}{|l|}{$\left(\right.$ Cells $\left./ \mathrm{mm}^{3}\right)$} \\
\hline $0-200$ & $247 / 3 \mid I(79.4 \%)$ & 1.00 & 1.00 \\
\hline $201-350$ & I59/176 (90.3\%) & $1.14(1.06-1.23)$ & $1.15(1.07-1.24)$ \\
\hline$>350$ & I58/I67 (94.6\%) & $1.19(1.11-1.27)$ & $1.17(1.09-1.26)$ \\
\hline
\end{tabular}

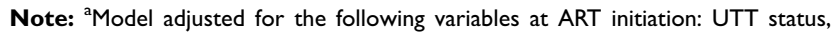
sex, age, and CD4 cell count.

Abbreviations: VL, Viral load; RR, Relative risk; UTT, Universal test and treat.

In this study, we were only able to observe treatment outcomes within the first 12 months of ART. The period immediately following first-line ART initiation may be unique as patients adjust to lifelong therapy. Consequently, a larger cohort with longer follow-up time is needed to identify longer-term outcomes, which may differ from the initial effects of treatment initiation up to 12 months on treatment. Moreover, follow-up data are ascertained primarily from routinely collected clinical data, the limitations of which, including missingness is well-documented and should be acknowledged here. ${ }^{39}$

Lastly, due to a lack of social and contextual data, our understanding of LTFU may be limited. Data relating to community and societal characteristics, income and social status, education level as well as stigma and barriers to treatment access are not routinely collected at the study sites. These factors are important to consider when developing a holistic understanding LTFU, ${ }^{40}$ and results presented here should be interpreted alongside these limitations.

\section{Conclusion}

While the implementation of universal test and treat in South Africa may ensure greater access to treatment among all known HIV-positive patients and increase viral suppression among those alive and in care 12 months after starting ART, rates of LTFU under these newly rolled-out guidelines prove higher than those of previous periods (even when restricted to only patients initiating ART with a CD4 cell count $\leq 500$ cells $/ \mathrm{mm}^{3}$ ). Patients initiating first-line therapy under the treat-all policy may often start treatment in better health, subsequently not perceiving a direct benefit to treatment. This coupled with the strict adherence protocol prescribed in first-line ART may deter patients from consistent engagement in HIV treatment programmes, while the need for sustained engagement with pre-ART programmes in order to initiate treatment, may lead to a more resilient pre-UTT population.

\section{Acknowledgments}

We extend our gratitude to the Directors and staff of Themba Lethu HIV Clinic and Witkoppen Health and Welfare Centre. We thank the Gauteng Provincial and National Department of Health for providing care to the patients at Themba Lethu HIV Clinic and Witkoppen Health and Welfare Centre as part of the National Programme. Finally, we sincerely thank the patients attending Themba Lethu HIV Clinic and Witkoppen Health and Welfare Centre for the use of their clinical data and their continued trust in the treatment and care provided at these clinics.

\section{Author Contributions}

All authors contributed to data analysis, drafting or revising the article, gave final approval of the version to be published, and agree to be accountable for all aspects of the work.

\section{Funding}

This study has been made possible by the generous support of the American people and the President's Emergency Plan for AIDS Relief (PEPFAR) through the United States Agency for International Development (USAID) under the terms of Cooperative Agreements AID-674-A-12-00029 and 72067419CA00004 to the Health Economics and Epidemiology Research Office. The contents are the responsibility of the authors and do not necessarily reflect the views of PEPFAR, USAID or the United States government. 


\section{Disclosure}

The authors declare that they have no competing interests.

\section{References}

1. Memirie S. Clinical outcome of children on HAART at police referral hospital, Addis Ababa, Ethiopia. Ethiop Med J. 2009;47(2):159-164.

2. Sawyer SM, Afifi RA, Bearinger LH, et al. Adolescence: a foundation for future health. Lancet. 2012;379(9826):1630-1640. doi:10.1016/S0140-6736(12)60072-5

3. Vreeman RC, Nyandiko WM, Ayaya SO, Walumbe EG, Marrero DG, Inui TS. The perceived impact of disclosure of pediatric HIV status on pediatric antiretroviral therapy adherence, child well-being, and social relationships in a resource-limited setting. AIDS Patient Care STDS. 2010;24(10):639-649. doi:10.1089/apc.2010.0079

4. Cohen MS, Chen YQ, McCauley M, et al. Prevention of HIV-1 infection with early antiretroviral therapy. $N$ Engl J Med. 2011;365 (6):493-505. doi:10.1056/NEJMoa1105243

5. Grinsztejn B, Hosseinipour MC, Ribaudo HJ, et al. Effects of early versus delayed initiation of antiretroviral treatment on clinical outcomes of HIV-1 infection: results from the Phase 3 HPTN 052 randomised controlled trial. Lancet Infect Dis. 2014;14(4):281-290. doi:10.1016/S1473-3099(13)70692-3

6. Insight Start Study Group. Initiation of antiretroviral therapy in early asymptomatic HIV infection. $N$ Engl J Med. 2015;373(9):795-807. doi:10.1056/NEJMoa1506816

7. The Strategies for Management of Antiretroviral Therapy (SMART) Study Group. Major clinical outcomes in antiretroviral therapy (ART)-naive participants and in those not receiving ART at baseline in the SMART study. $J$ Infect Dis. 2008;197(8):1133-1144. doi: $10.1086 / 588758$

8. Severe P, Jean Juste MA, Ambroise A, et al. Early versus standard antiretroviral therapy for HIV-infected adults in Haiti. $N$ Engl J Med. 2010;363(3):257-265. doi:10.1056/NEJMoa0910370

9. Statistics South Africa. Mid-Year Population Estimates. Pretoria, South Africa Africa. July 23 2018. Available from: http://www. statssa.gov.za/publications/P0302/P03022018.pdf. Accessed January 31, 2020.

10. Johnson LF, May MT, Dorrington RE, et al. Estimating the impact of antiretroviral treatment on adult mortality trends in South Africa: A mathematical modelling study. PLoS Med. 2017;14(12): e1002468. doi:10.1371/journal.pmed.1002468

11. Department of Health, Republic of South Africa. The South African National antiretroviral treatment guidelines 2004. Available from: http://southafrica.usembassy.gov/media/2004-doh-art-guidelines.pdf.

12. Department of Health, Republic of South Africa. The South African antiretroviral treatment guidelines 2013. Available from: http://www. sahivsoc.org/Files/2013\%20ART\%20Guidelines-Short\%20Combined \%20FINAL\%20draft\%20guidelines\%2014\%20March\%202013.pdf. Accessed January 31, 2020.

13. Department of Health, Republic of South Africa. National consolidated guidelines for the prevention of mother to child transmission of HIV (PMTCT) and the management of HIV in children, adolescents and adults 2015. Available from: http://www.sahivsoc.org/upload/ documents/ART\%20Guidelines\%2015052015.pdf.

14. Department of Health, Republic of South Africa. Implementation of the universal test and treat strategy for HIV positive patients and differentiated care for stable patients 2016 Available from: http:// www.sahivsoc.org/Files/22\%208\%2016\%20Circular\%20UTT\%20\% 20\%20Decongestion\%20CCMT\%20Directorate.pdf. Accessed January 31, 2020.

15. World Health Organization (WHO). Guideline on when to start antiretroviral therapy and on pre-exposure prophylaxis for HIV: World Health Organization; 2015. Available from: https://www.who.int/hiv/ pub/guidelines/earlyrelease-arv/en/. Accessed January 31, 2020.
16. Mberi MN, Kuonza LR, Dube NM, Nattey C, Manda S, Summers R. Determinants of loss to follow-up in patients on antiretroviral treatment, South Africa, 2004-2012: a cohort study. BMC Health Serv Res. 2015;15(1):259. doi:10.1186/s12913-015-0912-2

17. Joint United Nations Programme on HIV/AIDS (UNAIDS). 90-90-90 - an Ambitious Treatment Target to Help End the AIDS Epidemic. Geneva: UNAIDS; 2014. Available from: http://www.unaids.org/ sites/default/files/media_asset/90-90-90_en.pdf. Accessed January 31, 2020.

18. Fox MP, Maskew M, Brennan AT, et al. Cohort profile: the right to care clinical HIV cohort, South Africa. BMJ Open. 2017;7(6): e015620. doi:10.1136/bmjopen-2016-015620

19. Fox MP, Maskew M, MacPhail AP, et al. Cohort profile: the Themba Lethu Clinical Cohort, Johannesburg, South Africa. Int J Epidemiol. 2013;42(2):430-439. doi:10.1093/ije/dys029

20. Department of Health, Republic of South Africa. The South African Antiretroviral treatment guidelines 2010. Available from: http://www. sahivsoc.org/Files/Clinical_Guidelines_for_the_Management_of_ HIV_AIDS_in_Adults_Adolescents_2010.pdf. Accessed January 31, 2020.

21. Department of Health, Republic of South Africa. Circular on new criteria for initiating adults on ART at CD4 count of 350 cells $/ \mathrm{mL}$ and below [letter].

22. World Health Organization (WHO). Haemoglobin concentrations for the diagnosis of anaemia and assessment of severity. 2011. Available from: https://www.who.int/vmnis/indicators/haemoglo bin/en/. Accessed January 31, 2020.

23. Zou G. A modified poisson regression approach to prospective studies with binary data. Am J Epidemiol. 2004;159(7):702-706. doi:10.1093/aje/kwh090

24. Nglazi MD, Kaplan R, Wood R, Bekker L-G, Lawn SD. Identification of losses to follow-up in a community-based antiretroviral therapy clinic in South Africa using a computerized pharmacy tracking system. BMC Infect Dis. 2010;10(1):329. doi:10.1186/14712334-10-329

25. Rosen S, Fox MP, Gill CJ. Patient retention in antiretroviral therapy programs in sub-Saharan Africa: a systematic review. PLoS Med. 2007;4(10):e298. doi:10.1371/journal.pmed.0040298

26. Alvarez-Uria G, Naik PK, Pakam R, Midde M. Factors associated with attrition, mortality, and loss to follow up after antiretroviral therapy initiation: data from an HIV cohort study in India. Glob Health Action. 2013;6(1):21682. doi:10.3402/gha.v6i0.21682

27. Billong SC, Fokam J, Penda CI, et al. Predictors of poor retention on antiretroviral therapy as a major HIV drug resistance early warning indicator in Cameroon: results from a nationwide systematic random sampling. BMC Infect Dis. 2016;16(1):678. doi:10.1186/s12879-0161991-3

28. Geng EH, Bangsberg DR, Musinguzi N, et al. Understanding reasons for and outcomes of patients lost to follow-up in antiretroviral therapy programs in Africa through a sampling-based approach. J Acquir Immune Defic Syndr. 2010;53(3):405. doi:10.1097/QAI.0b013e3181b843f0

29. Loeliger KB, Niccolai LM, Mtungwa LN, Moll A, Shenoi SV. Antiretroviral therapy initiation and adherence in rural South Africa: community health workers' perspectives on barriers and facilitators. AIDS Care. 2016;28(8):982-993. doi:10.1080/09540121.2016.1164292

30. Mantell J, DiCarlo A, Remien R, et al. 'There's no place like home': perceptions of home-based HIV testing in Lesotho. Health Educ Res. 2014;29(3):456-469. doi:10.1093/her/cyu004

31. Van Cutsem G, Ford N, Hildebrand K, et al. Correcting for mortality among patients lost to follow up on antiretroviral therapy in South Africa: a cohort analysis. PLoS One. 2011;6(2):e14684. doi:10.1371/ journal.pone. 0014684

32. Miller CM, Ketlhapile M, Rybasack-Smith H, Rosen S. Why are antiretroviral treatment patients lost to follow-up? A qualitative study from South Africa. Trop Med Int Health. 2010;15:48-54. doi:10.1111/j.1365-3156.2010.02514.x 
33. Machado JK, Sant'Anna MJ, Coates V, Almeida FJ, Berezin EN, Omar HA. Brazilian adolescents infected by HIV: epidemiologic characteristics and adherence to treatment. Sci World J. 2009;9:1273-1285. doi:10.1100/tsw.2009.136

34. Mavedzenge SMN, Doyle AM, Ross DA. HIV prevention in young people in sub-Saharan Africa: a systematic review. J Adolescent Health. 2011;49(6):568-586. doi:10.1016/j.jadohealth.2011.02.007

35. Schwarz SW. Adolescent Reproductive and Sexual Health: Facts for Policymakers. 2010. http://www.nccp.org/publications/pub 931.html.

36. Brentlinger PE, Silva WP, Vermund SH, Valverde E, Buene M, Moon TD. Practical management of HIV-associated anemia in resource-limited settings: prospective observational evaluation of a new Mozambican guideline. AIDS Res Hum Retroviruses. 2016;32(1):12-25. doi:10.1089/aid.2015.0030

37. Riva E, Tettamanti M, Mosconi P, et al. Association of mild anemia with hospitalization and mortality in the elderly: the Health and Anemia population-based study. haematologica. 2009;94(1):22-28. doi:10.3324/haematol.13449
38. EuroCoord OIPTotCoOHERiEi. CD4 cell count and the risk of AIDS or death in HIV-Infected adults on combination antiretroviral therapy with a suppressed viral load: a longitudinal cohort study from COHERE. PLoS Med. 2012;9(3):e1001194. doi:10.1371/journal. pmed.1001194

39. Jorm L. Routinely collected data as a strategic resource for research: priorities for methods and workforce. Publ Health Res Pract. 2015;25(4):e2541540. doi:10.17061/phrp2541540

40. Dean HD, Fenton KA. Addressing Social Determinants of Health in the Prevention and Control of HIV/AIDS, Viral Hepatitis, Sexually Transmitted Infections, and Tuberculosis. Los Angeles, CA: SAGE Publications Sage CA; 2010.

\section{Publish your work in this journal}

Therapeutics and Clinical Risk Management is an international, peerreviewed journal of clinical therapeutics and risk management, focusing on concise rapid reporting of clinical studies in all therapeutic areas, outcomes, safety, and programs for the effective, safe, and sustained use of medicines. This journal is indexed on PubMed Central, CAS,
EMBase, Scopus and the Elsevier Bibliographic databases. The manuscript management system is completely online and includes a very quick and fair peer-review system, which is all easy to use. Visit http://www.dovepress.com/testimonials.php to read real quotes from published authors 